\title{
Development of a Cathodoluminescence Detection System for the (S)TEM Demonstrating Sub-nm Spatial and Sub-meV Spectral Resolution
}

\author{
David J Stowe ${ }^{1}$, Levi Beeching ${ }^{1,2}$, Richard J Vince ${ }^{1}$, John Oliver ${ }^{1}$, James Parsons ${ }^{1}$ and Simon A \\ Galloway $^{1}$ \\ 1. Gatan U.K. Abingdon, Oxon., United Kingdom \\ 2. Canberra, Australia
}

The development of nanomaterials with unique optical and electronic properties offers great potential in the advancement of optoelectronic devices, telecommunications and sensor technology. Cathodoluminescence (CL) microscopy in the SEM is a characterization technique which has enriched understanding of these materials through the correlation between morphology and luminescence, with spatial resolution approaching 10nm [1]. However, there remains a growing interest in performing CL microscopy in the (S)TEM where the morphology, microstructure and local chemistry can be attributed to the luminescence properties, at a spatial resolution potentially better than $1 \mathrm{~nm}[2,3]$.

Here we present a novel CL detection system suitable for a wide range of (S)TEM instruments and show exemplar results from semiconductor heterostructure nanorods and colloidal silver nanoparticles; luminescence information with a spatial resolution on the order of $1 \mathrm{~nm}$ is demonstrated.

Highly efficient collection of the CL signal is achieved through miniature diamond-turned mirrors integrated into the tip of a conventional cryogenic side-entry TEM holder. Mirrors above and below the specimen provide a solid angle of collection of up to 7.3steradian; a significant increase over existing systems where a single collecting mirror mounted to the TEM column is employed [4]. Light is coupled out of the holder through two NA-matched optical fibres to a Czerny-Turner optical spectrometer fitted with a photomultiplier tube and CCD providing a spectral resolution better than $0.5 \mathrm{~nm}(0.4 \mathrm{meV})$. In addition, the holder design is compatible with standard STEM imaging modes and allows the simultaneous acquisition of CL and EEL spectral data.

Figure 1 shows a nanorod with a periodic GaN/AlN quantum disc heterostructure with quantum disc nominal thicknesses of $2 \mathrm{~nm}$. A CL spectrum-linescan along the length of the nanorod clearly reveals the luminescence properties of many of the individual GaN quantum discs. Figure 2 shows two localized plasmon resonance modes excited by the primary electron beam of the TEM to be spatially and spectrally resolved by CL microscopy.

References:

[1] CE Norman, Proc. BIAMS 2000, Solid State Phenom. 78-79 (2000) p. 19

[2] SK Lim et al, Nano Letters 911 (2009) p. 3940

[3] LF Zagonel et al, Nano Letters 112 (2011) p. 568

[4] HP Strunk et al, J. of Microscopy 2241 (2006) p. 79 

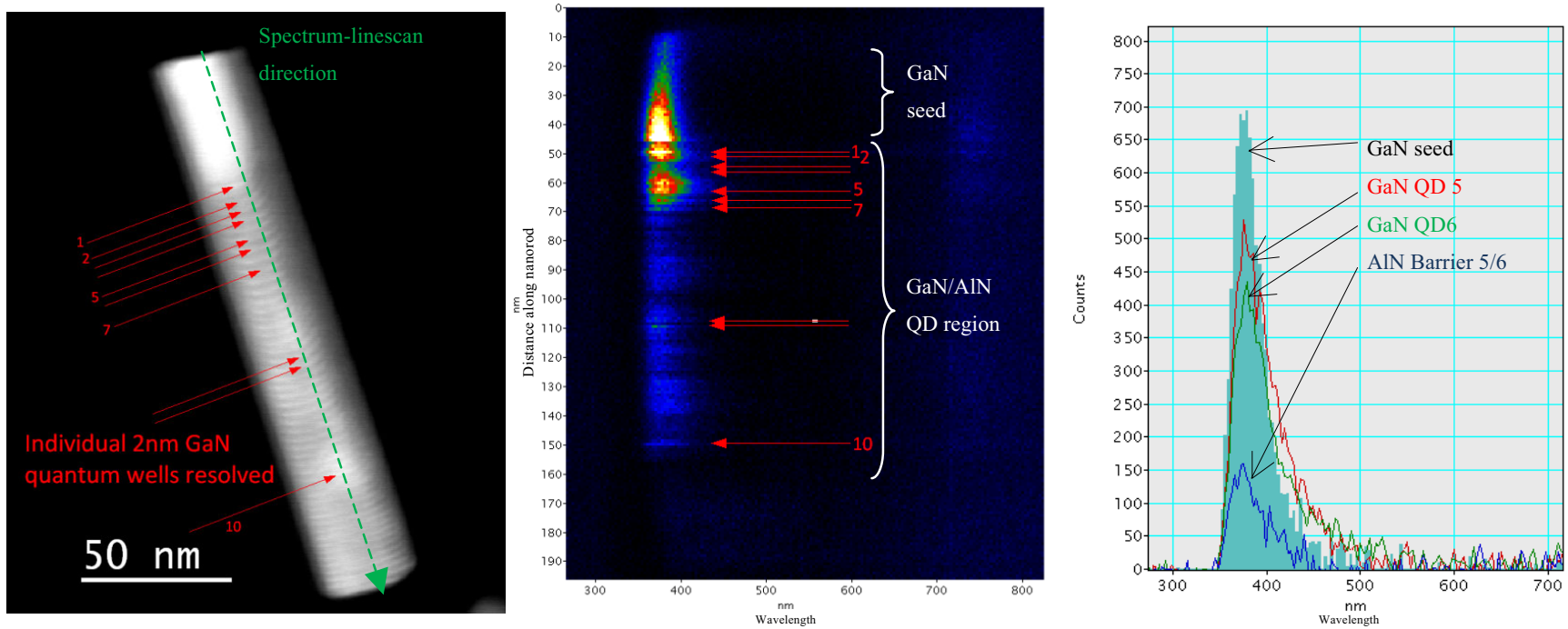

Figure 1. Left: HAADF STEM image of nanorod with GaN/AIN heterostructure (2nm quantum discs); GaN shows up bright. Centre: CL spectrum-linescan acquired along the length of the nanorod (indicated by the green line in the HAADF image); red arrows indicate quantum wells with directly attributable luminescence spectra; sample temperature: 90K. Right: selected CL spectra showing quantum discs 5 and 6 to be resolved spatially; specimen courtesy of Prof. R Myers, Ohio State University.
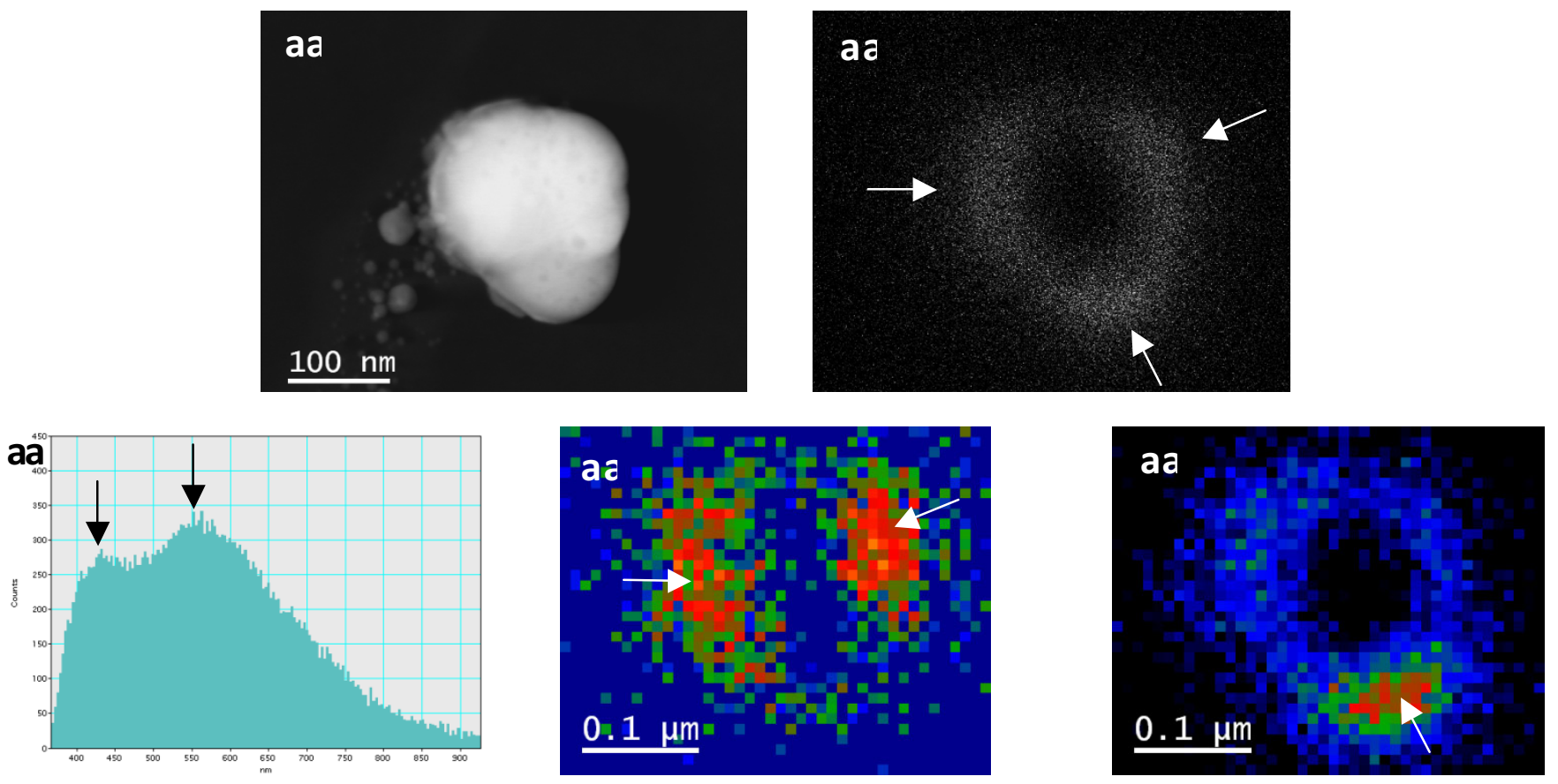

Figure 2. a) HAADF image of an egg-shaped colloidal silver nanoparticle; b) panchromatic cathodoluminescence image (acquired simultaneously to the HAADF image) displaying three 'bright' resonance nodes (indicated by arrow markers); c) cathodoluminescence spectrum with two peaks corresponding to spectrally discrete resonance modes at 430 and $510 \mathrm{~nm}$; d) and e) cathodoluminescence band pass images at 430 and $550 \mathrm{~nm} \pm 40 \mathrm{~nm}$ extracted from parent spectrum-image showing resonance modes are separated spatially and spectrally. 